\title{
Constrain the stellar population gradient of elliptical galaxies with SED
}

\author{
Zhengyi Shao \\ Key Laboratory for Research in Galaxies and Cosmology, Shanghai Astronomical Observatory, \\ CAS, Shanghai 200030, China \\ Key Lab for Astrophysics, Shanghai 200234,China \\ email: zyshao@shao.ac.cn
}

\begin{abstract}
We use multiple wavelength photometric data, from Optical to NIR, to determine the radial gradient of stellar populations of nearby elliptical galaxies. Empirical model, such as the Sersic profile, is assumed to describe the radial stellar distribution, and the metallicity gradient is parameterlized in this model. The PSF effects of different wavebands are also involved to predict the photometric profiles which can be compared with observational surface brightness profile directly. For most of elliptical galaxies, they have negative gradients, which means their central parts is metal rich than outer parts. We also found the gradient is significantly correlated to the stellar mass of the whole galaxy. More massive elliptical galaxies have shallower gradients. But for our sample galaxies, their is no correlations between Sersic $n$ and mass, and between Sersic $n$ and gradient can be found.
\end{abstract}

Keywords. galaxies: elliptical galaxies: photometry galaxies: abundances

\section{Introduction}

It is well-known that there exist the radial stellar population gradients in the early type galaxies (ETGs). This can be seen in the spectral absorption line indices and the shallow broad band color gradients. Although the spectral absorption line indices are the best tracers of the stellar population, the difficulties of the line observation confine the measurement within a range of one effective radius of galaxies. However, the measurement of the color gradient can extend to several effective radius or even farther. The moderate consistent of the dust free spectral absorption line indices gradient and the broadband color gradient indicate that photometric measurement is still one of the most important method to study the stellar population gradient in ETGs. It will give restrictions on the model of galaxy formation and evolution.

The Sloan Digital Sky Survey (SDSS) and the Two Micron ALL Sky Survey (2MASS) produce large and homogeneous databases and high quality images in five optical and three near infrared bands. This provides an opportunity to study the stellar population gradients with a large sample of nearby galaxies. The spectral energy distribution (SED) from the optical to NIR, at different radius will give us much more information about the stellar population distribution than before. Thus we can quantitatively constrain stellar population gradients of individual galaxies.

\section{Sample galaxies and photometric data}

Nair \& Abraham (2010) classified SDSS(DR4) galaxies with $g<16.0$ and $0.01<$ $z<0.1$ by naked eye. We further select brighter $(g<15.0)$ ETGs $(T<0)$ from their 
sample, and matched them with 2MASS extend sources. Finally, we got 2123 ETGs with photometric data of eight broad wavebands from optical to NIR (ugrizJHK $K_{s}$ ). Photometric profiles are used in this work. For SDSS, we use profmean values, and for 2MASS we use round aperture magnitudes. Typically, the outer most SDSS annulus and 2MASS aperture can reach 30 and 50 arcsec respectively. It is almost $6 \sim 10$ times as large as the effective radius of our sample galaxies.

\section{Method}

In this work, we introduce a new approach to modelling the galaxy profile. Instead of fitting galaxies in different bands separately, we tried to combine observational data of all given wave bands.

We assume the stellar column density profile (not the surface brightness of a single band as commonly used before) of an ETG follows the Sersic profile. Giving a set of parameters, such as stellar mass $\left(M_{\text {star }}\right)$, Sersic index $(n)$, effective radius $\left(R_{e}\right)$, metallicity $\left(Z_{m e t}\right)$ and age $(t)$, and use single stellar population (SSP) model of synthesized spectra (BC03), we can predict the observational profiles in each band, such as profmens of SDSS and aperture magnitudes of 2MASS. Using this method, we can fit these parameters simulatively. Also, an additional parameter should be involved to describe the gradient of stellar population. For simplicity, we only use the radial metallicity gradient $\left(g_{Z} \equiv \Delta \log Z_{m e t} / \Delta \log R\right)$ to represent all gradient effects. Because as Wu et. al. (2005) concluded that metallicity dominates the radial gradients of stellar population of ETGs.

During the fitting, PSFs of different bands are all considered in calculating their surface brightness profiles separately. The axis ratio of isophote at $g$ band is also involved to correct the elliptical shape effect of the galaxy.

\section{Results}

Fig. 1 shows our fitting results of ETG's shape of stellar column density $(n)$ and metallicity gradient. Sersic index $n$ are mainly distributed from 3.1 To 4.7, with median value of 3.8, which pretty well appears as de Vaucouleurs shape. Radial metallicity gradients are distributed from -0.49 to -0.03 , with median value of -0.24 . This is in good agreement with $\mathrm{Wu}$ et. al. (2005). There are $12 \%$ sample galaxies have positive gradients, corresponding to the bluer core ETGs. This could be due to a recent star forming in the central region, though we only assume a metallicity gradients.

In Fig. 2, we can find a significant correlation between stellar mass and radial gradient. It shows more massive ETGs have shallower gradients. Also, form this figure, we can find
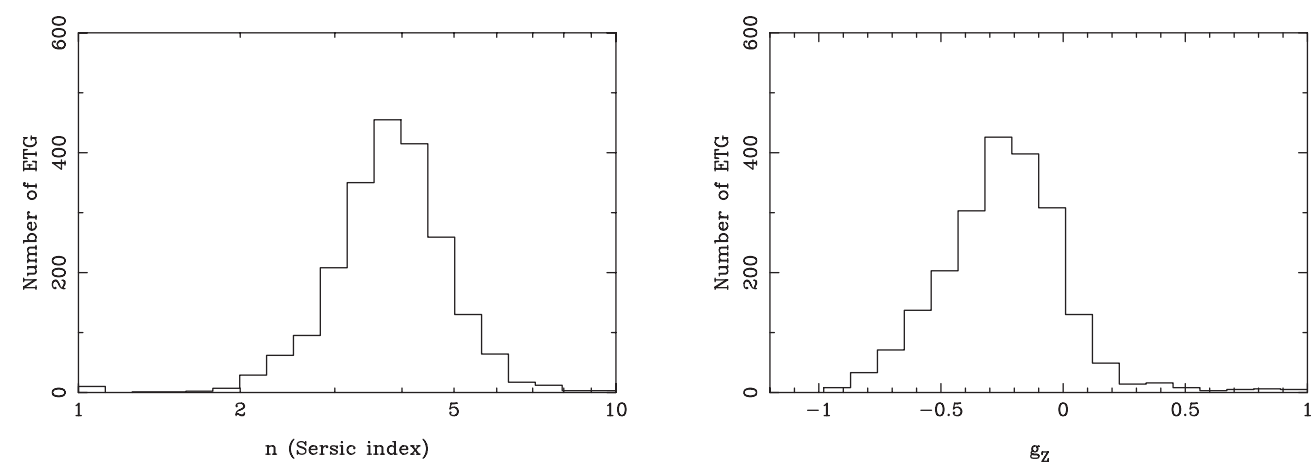

Figure 1. Distribution of fitted Sersic index $n$ and metallicity gradient of ETGs 


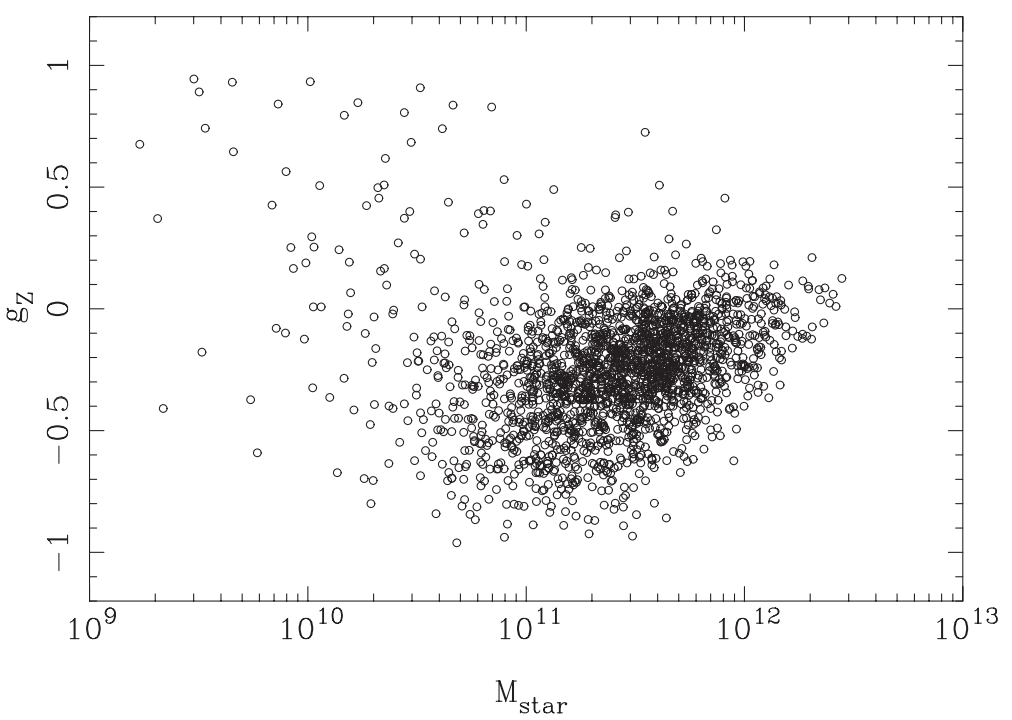

Figure 2. Correlation between stellar mass and metallicity gradient

most of the galaxies with positive value of gradients are smaller ones, and they do not follow this relationship of bigger galaxies.

\section{Discussions}

Our results confirm that only assume the stellar column density profile of ETGs, their observational surface brightness profile can be well predicted. The radial distribution of stellar mass can also be well modelled by Sersic profile, and their radial gradient of stellar population can be constrained.

The scatter of $g_{Z}, \sigma_{g_{z}} \geqslant 0.2$ is relatively large that can not be only explained by the uncertainties of photometric measurement or fitting approach. So, the scatter must be intrinsic. The formation process such as major and minor merger may all play as important roles, and the environment of these galaxies are also need to be taken into account.

An significant correction between stellar mass and radial gradient is found for most of our sample galaxies, except for those smallest ETGs with positive gradient. There is no correction between stellar mass and Seraic $n$, and no correction between gradient and $n$. These all imply that the mass is the most dominated factor during the ETG formation and evolution process.

\section{References}

Bruzual G. \& Charlot S. 2003, MNRAS, 344, 1000

Nair, P. B. \& Abraham, R. G. 2010, ApJS, 186, 427

Wu H., Shao Z. et al. 2005, ApJ, 622, 244 